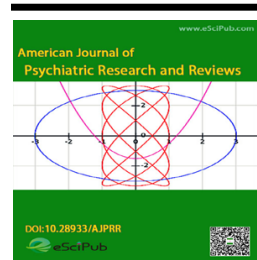

American Journal of Psychiatric Research and Reviews (ISSN:2637-479X)

\title{
COVID-19 Lockdown Fatigue
}

\section{Tiffany Field ${ }^{12^{*}}$, Shantay Mines ${ }^{2}$, Samantha Poling ${ }^{2}$, Miguel Diego $^{1}$, Debra Bendell ${ }^{2}$, and Connie Veazey ${ }^{2}$}

${ }^{1}$ University of Miami/Miller School of Medicine. ${ }^{2}$ Fielding Graduate University.

\begin{abstract}
In this COVID-19 lockdown Survey Monkey study, as many as 75\% of 260 respondents reported feeling fatigued. Correlation analyses suggested that feeling fatigued was significantly correlated with demographic variables and virtually every item on every scale of the survey. The demographic correlations suggested that fatigue occurred more frequently in younger participants, in males, and in those not working from home during the lockdown. The significant correlations for the scales suggested that those feeling fatigued engaged in fewer health activities including exercise and self-care; they spent more time on social media including gaming and Facebook; they engaged in less cooking and creative projects; they scored higher on the Stress Scale including worrying more about the virus and their finances; they reported feeling more isolated, lonely, bored and touch deprived; they did more snacking and napping and expressed more "cabin fever"; they had lower scores on connecting and activities at home; and they had higher scores on anxiety, depression, sleep disturbances, and PTSD scales. A regression analysis suggested that $51 \%$ of the variance in the fatigue scores was explained by the depression (37\% variance), sleep disturbances $(12 \%)$ and anxiety (1\%) scores. These results are limited by their being self-reported data from a non-representative, crosssectional sample. Nonetheless, they highlight the negative effects of feeling fatigued during a COVID-19 lockdown.
\end{abstract} *Correspondence to Author: Tiffany Field

University of Miami/Miller School of Medicine.

Keywords: COVID-19 lockdown; Fatigue; Sleep disturbances; Anxiety; Depression

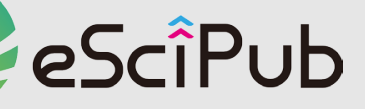

eSciPub LLC, Houston, TX USA. Website: https://escipub.com/ 
Fatigue has received very little attention in the COVID-19 literature, although, anecdotally, it has been frequently expressed during lockdowns and especially after several months of the pandemic when it has been increasingly referred to as "pandemic fatigue". Only a few publications could be found on fatigue in the COVID-19 literature.

One research group used the Daily Life Fatigue Scale that included items on physical, mental and social fatigue. ${ }^{[1]}$ In this survey from Poland, women and those who were living alone experienced the most fatigue. However, $85 \%$ of the sample was female, and females had less exercise prior to the COVID-19 lockdown which may have contributed to their fatigue. That those living alone experienced more fatigue was not surprising as those living alone during COVID-19 in the U.S. have reported greater isolation and loneliness which may have explained their fatigue. [2] Unfortunately, the authors of the study from Poland did not compare the different types of fatigue that were tapped by their scale including physical, mental, and social fatigue. And, as in any survey, access to the internet and self-selection for responses to internet surveys limited these results.

In a second COVID-19 fatigue study, fatigue was referred to as a reluctance to undertake physical, mental and social activities, and it was said to derive from pandemic-related fear and anxiety. [3] Other references to COVID-19 fatigue have included media fatigue as in Facebook fatigue in a study from Finland ${ }^{4]}$ and research fatigue from participating in many online surveys during the COVID-19 pandemic. [5]

Fatigue has also rarely appeared in the preCOVID-19 literature except as it relates to sleep deprivation, post-sports event fatigue, or caregiver fatigue. There, it has been typically defined as a vague feeling of being tired, weak or exhausted. Fatigue has not been a primary focus but is sometimes listed as a symptom of depression, anxiety, and sleep problems or resulting from sedentary activity and the lack of exercise. The COVID-19 literature on psychological problems has focused primarily on stress, anxiety, depression, sleep disturbances, and PTSD. [6-11] And, fatigue has rarely been studied as a psychological problem or even as a correlate of these lockdown problems. The purpose of the present data analyses was to assess the relationships between Fatigue Scale scores and scores on scales of health activities, stress, anxiety, depression, sleep disturbances, and PTSD symptoms in individuals experiencing a COVID19 lockdown. Fatigue was expected to be associated with other COVID-related problems including stress, anxiety, depression, sleep disturbances and PTSD symptoms.

\section{Methods}

\section{Participants}

$A G^{*}$ power analysis indicated that a sample size of 224 was required for an alpha of .05 and $80 \%$ power. The participants included individuals $(\mathrm{N}=260)$ who ranged in age from 18-82 ( $\mathrm{M}=47$ years). Gender was distributed $79 \%$ female, $18 \%$ male and $3 \%$ other (non-specified). Ethnicity was distributed $68 \%$ Non-Hispanic White, $21 \%$ Hispanic, 3\% Black and $8 \%$ other (nonspecified). Professions were distributed $35 \%$ office worker, 30\% academic, 15\% managerial, $12 \%$ medical and $8 \%$ labor. The average income was $\$ 72,572,28 \%$ were unemployed and $69 \%$ worked at home. Twenty-three per cent lived alone.

\section{Procedure}

A flyer was posted on Facebook giving a brief description of the study including some sample items and the age criterion being greater than 18 years. The Facebook flyer included a link to the survey on Survey Monkey which included 11 scales for a total of 87 items. The survey was four weeks duration (April 1-30, 2020), and the data were directly transported to SPSS for data analyses.

\section{Measures}

The survey included several demographic items including those already mentioned (age, gender, ethnicity, profession, income, type of employment, working at home, and living alone). The following five scales were created specifically for this survey to relate to activities and stress associated with the COVID-19 lockdown. [12] The 
participants rated the items on the scales from zero meaning "not at all" to three meaning "a lot" including the:

1) Health Scale (15 items) (Cronbach's alpha $=.66$ ) which included exercise (inside exercise, outside exercise and outside exercise with others as well as the types of exercise), touching (touching partner, kids and self as well as the types of touching), COVID- 19-related safety practices including washing hands and social distancing, self-care, spiritual activities (meditating and feeling spiritual), and liking being at home. A factor analysis yielded three factors contributing to $47 \%$ of the variance on the Health Scale score: Factor 1 Self/Spiritual Care that included Meditating (.74), Self-Care (.68), and Feeling Spiritual (.77) items that together explained $23 \%$ of the variance; Factor 2 Touching that included the items Touching your kids (.75) and Touching your partner or friend (.72) that explained $14 \%$ of the variance; and Factor 3 Exercise that included the items Outside exercise (-.89) and Exercise outside with someone else (-.76) that explained $10 \%$ of the variance;

2) Media/Communications Scale (10 items) (Cronbach's alpha=.58) including talking on the phone, texting, on Internet, gaming, on Facebook/Instagram, spending time receiving and sending messages/media about the virus, engaging in Zoom/Skype/Facetime activities (e.g. Yoga, meditation), watching the news, watching other TV programs, and watching movies. A factor analysis yielded four factors contributing to $61 \%$ of the variance on the Media/Communication Scale score: Factor 1 Entertainment that included the items Watching movies (.84) and TV programs $(.80)$ that explained $23 \%$ of the variance; Factor 2 Communication that included phone use (.80), texting (.70) and Zoom (.63) that explained $14 \%$ of the variance; Factor 3 Social Media that included being on internet (.78) and Facebook time (.60) that explained $13 \%$ of the variance; and Factor 4-COVID News that included watching the news (.79) and messaging about the virus (.60) that explained $11 \%$ of the variance;

3) Connecting Scale (4 items) (Cronbach's alp- $\mathrm{ha}=.41$ ) which included connecting with friends, trying to connect with old friends, helping children do homework, and receiving support from others;

4) Working Scale (6 items) (Cronbach's alpha=.61) including cooking, caregiving, housekeeping, paperwork, creative work, and working on projects/hobbies; and

5) Stress Scale (11 items) (Cronbach's alpha $=.78$ ) which included worrying about getting a virus, worrying about your financial status, wanting this experience to end, feeling isolated, feeling lonely, feeling bored, feeling touch deprived, snacking, drinking alcohol, napping, and getting "cabin fever". A factor analysis yielded three factors contributing to $56 \%$ of the variance on the Stress Scale score: Factor 1 Stimulation deprivation that included the items Feeling Isolated (.86), Feeling lonely (.86), Feeling bored (.74), Getting cabin fever (.70), and Feeling touch deprived (.65) that together explained $34 \%$ of the variance; Factor 2 Worrying that included the items Worried about finances (.67) and Worried about the virus (.47) that explained $12 \%$ of the variance; and Factor 3 Stress behaviors that included the items Napping (.68) and Snacking (.53) that explained $10 \%$ of the variance.

The standardized scales on the survey included 4 PROMIS Subscales ${ }^{13}$ (each item was rated on a 5 -point scale as $1=$ never, $2=$ rarely, $3=$ sometimes, $4=$ often, and $5=$ always) which included the: 1) PROMIS Anxiety Subscale (4 items) (Cronbach's alpha=.88) which included I felt fearful, I found it hard to focus on anything other than my anxiety, my worries overwhelmed me, and I felt uneasy;

2) PROMIS Depression Subscale (4 items) (Cronbach's alpha=.91) including I felt worthless, helpless, depressed, and hopeless;

3) PROMIS Fatigue Subscale (3 items) (Cronbach's alpha $=.92$ ) including I felt fatigued, I had trouble starting things because I'm tired, and I felt run-down; and

4) PROMIS Sleep Disturbance Subscale (4 items) (Cronbach's alpha $=.86$ ) which included my sleep quality was bad, my sleep is not AJPRR: https://escipub.com/american-journal-of-psychiatric-research-and-reviews/ 
refreshing, I had a problem with my sleep, and I had difficulty falling asleep.

The second standardized scale was a PTSD Screener entitled "PTSD-8: A short PTSD Inventory" (8 items) (Cronbach's alpha $=.92) .{ }^{14}$ This inventory is introduced by the statement "If you're being reminded of a traumatic experience, please rate how much the following have bothered you during the lockdown" as: 0) not at all, 1) rarely, 2) sometimes, and 3) most of the time. The items are: recurrent thoughts and memories of the event, feeling as though the event is happening again, recurrent nightmares about the event, sudden emotional or physical reactions when reminded of the event, avoiding activities that remind you of the event, avoiding thoughts or feelings associated with the event, feeling jumpy/easily startled, and feeling on guard.

\section{Results}

\section{Correlation Analyses Yielding Significant Coefficients for Fatigue Scale Scores}

Results indicated that $75 \%$ of 260 respondents reported feeling fatigued, including $22 \%$ who rated themselves as being "a little" fatigued (a rating of 2), $30 \%$ as feeling somewhat fatigued (a rating of 3 ), $23 \%$ as feeling fatigued "quite a lot" (a rating of 4 ), and $10 \%$ rating "very much" fatigue (a rating of 5). Correlation analyses revealed a number of significant correlation coefficients for the Fatigue Scale Scores (at the $p<.05$ level) (see Table 1 for the correlation coefficients for the scales' total scores) including: 1) several demographic variables suggested that Fatigue Scale scores were higher for younger participants, for males, and for those not working at home;

Table 1. Correlation coefficients for significant relationships between feeling Fatigue Scale scores and scores on other COVID-19 Lockdown Survey scales and subscales.

\begin{tabular}{lcl} 
Measure & Correlation coefficient & p level \\
\hline Health Scale Score & -.35 & .0001 \\
Connecting Scale Score & -.17 & .009 \\
Working Scale Score & -.14 & .03 \\
Stress Scale Score & .49 & .0001 \\
PROMIS Anxiety Subscale Score & .62 & .0001 \\
PROMIS Depression Subscale Score & .64 & .0001 \\
PROMIS Sleep Disturbance Subscale Score & .63 & .0001 \\
PTSD-8 Inventory Score & .48 & .0001 \\
\hline
\end{tabular}

2) negative correlations with the Health Scale total score and its items indicated less inside exercise, outdoor exercise, outdoor exercise with someone else, self-care, feeling spiritual and "liking being at home"; 3) a negative correlation with the Connecting Scale total score; 4) a negative correlation with the Working Scale total score as well as less cooking and less working on creative projects; 5) positive correlations with the Media/Communication Scale total score and its items including more gaming, Facebook time, and more messaging about the virus; 6) positive correlations with the Stress Scale total score and several items suggesting more worrying about the virus and about finances, feeling more isolated, lonely, bored, and touch deprived, more snacking and napping, and experiencing more "cabin fever; 7) positive correlations with the PROMIS Anxiety Subscale score and all its items including feeling fearful, having difficulty focusing on anything other than my anxiety, worries overwhelming me and feeling uneasy; 8) positive correlations with the PROMIS Depression Subscale total score and all its items including feeling worthless, helpless, depressed and hopeless; 9) positive correlations with the PROMIS Fatigue Subscale items including fatigue, tired, and rundown; 10) positive correlations for the PROMIS Sleep Disturbance Subscale total score and all its items including sleep quality, refreshing sleep, problem sleeping, and difficulty falling asleep; and 10) positive 
correlations for the Posttraumatic Stress Inventory-8 total score and all of its items including thoughts or memories of the event, feeling that the traumatic experience is happening again, having nightmares about the event, sudden emotional or physical reactions when reminded of the event, avoiding activities that remind you of the event, avoiding thoughts or feelings associated with the event, feeling jumpy/easily startled, and feeling on guard.

Regression Analyses on Scales Significantly Related to Fatigue Scale Scores

Two stepwise regression analyses were conducted to determine: 1) in the first regression, the amount of the variance in the Fatigue Scale scores that was explained by the psychological problem scale scores that were moderately to highly significantly related to the Fatigue Scale scores including the Stress Scale $(r=.49)$, the PROMIS Anxiety Subscale ( $r=.62)$, the PROMIS Depression Subscale $(r=.64)$, the PROMIS Sleep Disturbances Subscale $(r=.63)$ and the PTSD-8 Inventory ( $r=.48)$ scale scores; and 2$)$ in the second regression, the amount of variance in the Fatigue Scale scores that was explained by the activity scale scores that were significantly related to the Fatigue Scale scores including the Health Scale ( $r=-.35)$,the Connecting Scale $(r=-.17)$, and the Working Scale $(r=-.14) \mathrm{s}$ scores.

In the first stepwise regression in which the psychological problem scores were entered, the PROMIS Depression Subscale score explained $37 \%$ of the variance, the PROMIS Sleep Disturbances Subscale score added $12 \%$ of the variance and the PROMIS Anxiety Subscale score added another $1 \%$ to the variance for a total of $51 \%$ of the variance in the PROMIS Fatigue Subscale scores that was explained by the three other scores (see Table 2 for the regression analyses). In the second stepwise regression in which the activity scores were entered, only the Health Scale score was entered which explained only $11 \%$ of the variance on the PROMIS Fatigue Subscale scores.

\section{Table 2. Stepwise regression analyses on Fatigue Scale scores:}

a) entering significantly related psychological problem scale scores including stress, anxiety, depression, sleep disturbances, and PTSD symptoms

\begin{tabular}{lccccc} 
Scale & $\mathrm{R}$ & $\mathrm{R}^{2}$ & $\mathrm{R}^{2}$ change & $\mathrm{F}$ change value & $\mathrm{plevel}$ \\
\hline Depression & .61 & .37 & .37 & 102.92 & .0001 \\
Sleep Disturbances & .70 & .49 & .12 & 40.89 & .0001 \\
Anxiety & .71 & .51 & .01 & 5.02 & .0001 \\
\hline
\end{tabular}

b) entering significantly related activity scale scores including health, connecting, and working

\begin{tabular}{llcccc} 
Scale & $R$ & $R^{2}$ & $R^{2}$ change & F change value & $p$ level \\
\hline Health & .33 & .11 & .11 & 26.23 & .0001 \\
\hline
\end{tabular}

Table 3. Mean scale scores for significant ANOVAs for low versus high Fatigue Scale score groups (standard deviations in parentheses) based on a median split.

\begin{tabular}{lccccc} 
Measure & Low & High & F value & p level & eta $^{2}$ \\
\hline Health & $33.95(5.30)$ & $29.95(5.35)$ & 20.28 & .0001 & .08 \\
Connecting & $9.05(2.47)$ & $8.62(2.06)$ & 6.30 & .01 & .03 \\
Stress & $23.82(5.12)$ & $30.18(5.90)$ & 58.20 & .0001 & .19 \\
Anxiety & $7.82(2.76)$ & $11.62(3.30)$ & 103.03 & .0001 & .29 \\
Depression & $6.09(2.38)$ & $10.63(3.95)$ & 121.87 & .0001 & .32 \\
Sleep Disturbance & $11.27(3.86)$ & $16.39(3.81)$ & 74.55 & .0001 & .23 \\
PTSD & $11.66(4.74)$ & $16.48(5.67)$ & 31.29 & .0001 & .15 \\
\hline
\end{tabular}

Analyses of Variance on a Median Split of the Fatigue Scale Scores

Analyses of variance were conducted to provide confirmatory evidence for the correlation and regression analyses. As can be seen in table 3 , the ANOVAs on the median split significantly differentiated the groups on all the scale scores except the media scale score which was consistent AJPRR: https://escipub.com/american-journal-of-psychiatric-research-and-reviews/ 
with the correlation and regression analyses results. A MANOVA on the Fatigue Scale scores was significant (Wilks' Lambda $F=11.72$, $p=.0001$ ).

\section{Discussion}

Although fatigue has been less frequently studied than depression, anxiety, and sleep disturbances during COVID-19 lockdowns, [6-11] the data from the current survey highlight the prevalence of fatigue (75\%). That Fatigue Scale scores were positively correlated with virtually every negative item of every scale and negatively related to every positive item, highlights the negative effects of fatigue during lockdowns. Its positive correlations with other psychological problems including stress, anxiety, depression, sleep disturbances, and PTSD symptoms suggest that it needs to be studied together with those problems that have received more attention in the COVID-19 lockdown literature. ${ }^{[6-11]}$ The stepwise regression suggested that $51 \%$ of the variance in fatigue could be accounted for by depression (37\%), sleep disturbances (12\%), and anxiety $(1 \%)$. That anxiety accounted for so little variance in fatigue was surprising given the earlier COVID-19 study that ascribed fatigue to fear and anxiety about the pandemic. ${ }^{[3]}$

The directionality of these data cannot, of course, be determined in a cross-sectional survey like this. Whether depression, anxiety and sleep disturbances contributed to fatigue, suggesting that fatigue is a symptom of these problems or that fatigue contributed to these mood states is not clear. Fatigue and these mood states may be bi-directional. At the very least, fatigue might be studied alongside these problems or maybe as an early sign of the more serious problems of anxiety, depression and sleep disturbances.

Other scores that were highly related to fatigue included scores on the Stress Scale and the PTSD inventory. PTSD symptoms have been noted in other lockdown literature as well as in this COVID-19 sample. ${ }^{[15]}$ The stressors that contributed to most of the variance in the Stress Scale scores included feeling isolated and lonely. These are not surprising as they have been noted in other COVID-19 data analyses as being related to fatigue. ${ }^{[2]}$

Other Stress Scale items that were highly correlated with fatigue including feeling bored and touch deprived. Although those problems have been highlighted in other papers on this lockdown survey, they have typically not been associated with fatigue. $[12,16]$

The lack of exercise has also been highlighted as a COVID-19 lockdown problem. ${ }^{[17]}$ The current data analyses suggest that the more fatigued engaged in less indoor exercise, less outdoor exercise and less outdoor exercise with someone else. These negative relationships highlight the possibility that exercise may ameliorate the effects of fatigue.

Other activities that were notably related were not working from home, not cooking and not engaging in creative projects. These activities may buffer COVID-related fatigue. The relations between fatigue and Facebook time and gaming are consistent with pre- COVID literature that refers to Facebook fatigue or social media fatigue as well as COVID-19 studies suggesting that some social media activities are positive such as texting, but others are mixed such as internet and some are negative such as Facebook time. [18]

The demographic variables that were related to fatigue were surprising, i.e. being younger and male gender. Being younger is surprising because fatigue is usually associated with aging, and being male and feeling fatigue is inconsistent with at least two previous COVID studies that found more fatigue in females. ${ }^{[1,3]}$ The younger participants may have experienced more fatigue related to living alone which is consistent with previous data analyses on being young and living alone ${ }^{[19]}$ as well as being related to feeling isolated and lonely. ${ }^{[2]}$ The male gender finding may relate to men engaging in less cooking and creative projects and/or engaging in less work from home.

Those activities that were negatively correlated with Fatigue Scale scores could be considered buffers to fatigue including working from home and engaging in indoor exercise, outdoor 
exercise and outdoor exercise with someone else as well as self-care, cooking, and creative projects. The scales that assessed these activities including the Health, Connecting and Working scales were entered into a stepwise regression on the Fatigue Scale scores. Although significant, this analysis, as noted, only explained $11 \%$ of the variance, with Health Scale scores (that included exercise, touching, and meditation items) being the only variable that entered the analysis. The relationships between fatigue and inactivity in this sample is consistent with the reference to fatigue as "a reluctance to undertake physical, mental and social activities". [3] Fatigue was a deterrent to most activities in this sample. Methodological limitations of these data included sampling and assessment issues. Being a predominantly non-Hispanic, white female sample suggests that these data may not be generalizable to the larger population. The self-report data are subject to questionable bias and reliability, although their anonymity suggests that they may have not been falsely reported. The direction of the effects, i.e. the predictive value of depression and sleep disturbances for fatigue cannot be determined given that the data are cross-sectional rather than longitudinal. As in most other studies in the COVID-19 psychological problems literature, no baseline data were available. And, the unpredictable duration of the lockdown as well as the anonymity of the survey precluded the collection of longitudinal data. However, another analysis of these data compared a mid-term sample to the larger end-of-study sample. ${ }^{[2]}$ That analysis suggested that all the psychological problems that were significantly related to fatigue in the current data analysis were experienced by a greater percent of the sample by the end of the study including anxiety, depression, and sleep disturbances. Those increases ranged from 23 to $57 \%$. And the Stress Scale problems increased from 40 to $62 \%$ including feeling isolated, lonely, touch deprived, and cabin fever. Feeling fatigue increased by $56 \%$. But, at least one of the fatigue buffers, i.e. exercise, fortunately increased by $86 \%$.
Despite these methodological limitations, these survey data suggest that fatigue was a significant lockdown problem in this sample. Fatigue was most related to stressors including feeling isolated, lonely, and touch deprived and having cabin fever. That depression and sleep disturbance scores explained a moderate amount of the variance on fatigue suggests that at least for this sample, depression and sleep disturbances were contributing to the high prevalence of fatigue. Although a small amount of the variance in fatigue was explained by the lack of health activities, that finding is suggestive that lockdown fatigue could be alleviated by health activities like exercise, touching, and meditation. Laboratory and longitudinal studies may further inform the literature on fatigue as well as interventions that may alleviate the fatigue associated with lockdowns like those of the COVID-19 pandemic.

\section{References}

[1] Bartoszek, A., Walkowiak, D., Bartoszek, A.\& Kardas, G. Mental Well-Being (Depression, Loneliness, Insomnia, Daily Life Fatigue) during COVID-19 Related Home-Confinement-A Study from Poland Int. J. Environ. Res. Public Health 2020, 17, 7417; doi:10.3390/ijerph 17207417

[2] Field, T., Poling, S., Mines, S., Bendell, D. \& Veazey, C. Feeling isolated and lonely during a COVID-19 lockdown. Archives of Health Science. 2020, 4 (1), 1-9.

[3] Morgul, E.; Bener, A.; Atak, M.; Akyel, S.; Aktas, S.; Bhugra, D.; Ventriglio, A.; Jordan, T.R. COVID-19 pandemic and psychological fatigue in Turkey. Int. J. Soc. Psychiatry 2020

[4] Islam,N, , Laatob, S, Talukderc, S. \& Sutinen, E. Misinformation sharing and social media fatigue during COVID-19: An affordance and cognitive load perspective. Technological Forecasting and Social Change, 2020, 159

[5] Weston, D., \& Brooks, S. Research fatigue in COVID-19 pandemic and post-disaster research: causes, consequences and recommendations. Disaster Prevention and Management, 2020

[6] Huang Y, Zhao N. Generalized anxiety disorder, depressive symptoms and sleep quality during COVID-19 outbreak in China: a web-based cross-sectional survey. Psychiatry Res. 2020 Jun; 288:112954. doi: 10.1016/j.psychres. 2020. 
112954. Epub 2020 Apr 12. PMID: 32325383; PMCID: PMC7152913.

[7] Wang C, Pan R, Wan X, Tan Y, Xu L, Mclntyre RS, Choo FN, Tran B, Ho R, Sharma VK, Ho C. A longitudinal study on the mental health of general population during the COVID-19 epidemic in China. Brain Behav Immun. 2020 Jul;87:40-48. doi: 10.1016/j.bbi.2020.04.028. Epub 2020 Apr 13. PMID: 32298802; PMCID: PMC7153528.

[8] Altena E, Baglioni C, Espie CA, Ellis J, Gavriloff D, Holzinger B, Schlarb A, Frase L, Jernelöv S, Riemann D. Dealing with sleep problems during home confinement due to the COVID-19 outbreak: Practical recommendations from a task force of the European CBT-I Academy. J Sleep Res. 2020 Apr 4:e13052. doi: 10.1111/jsr. 13052. Epub ahead of print. PMID: 32246787.

[9] Li J, Yang Z, Qiu H, Wang Y, Jian L, Ji J, Li K. Anxiety and depression among general population in China at the peak of the COVID-19 epidemic. World Psychiatry. 2020 Jun;19(2):249250. doi: 10.1002/wps.20758. PMID: 32394560; PMCID: PMC7214959.

[10] Liu N, Zhang F, Wei C, Jia Y, Shang Z, Sun L, Wu L, Sun Z, Zhou Y, Wang Y, Liu W. Prevalence and predictors of PTSS during COVID-19 outbreak in China hardest-hit areas: Gender differences matter. Psychiatry Res. 2020 May; 287:112921. doi: 10.1016/j.psychres.2020. 1129 21. Epub 2020 Mar 16. PMID: 32240896; PMCID: PMC7102622.

[11] Forte, G., Faviori, F., Tambelii, R. \& Casagrande, M. COVID-19 pandemic in the Italian population: Validation of a post-traumatic stress disorder questionnaire and prevalence of PTSD symptomatology. International Journal of
Environmental Research in Public Health, 2020, 17, 4151.

[12] Field, T., Mines, S., Poling, S., Bendell, D. \& Veazey, C. Touch deprivation and exercise. Medical research Archives. 2020, 8 (8), 1-12.

[13] Dewitt, B., Feeny, D., Fischhoff, B., Celia, D., Hays, R.D. et al. Estimation of a preferencebased summary score for the patient reported outcomes measurement information system: The PROMIS-preference (PROPr) scoring system. Medical Decision Making. 2018: 38: 683698.

[14] Hansen, M., Anderson, T. E., Armour, C., Elklit, A., Palic,S., \& Mackrill, T. PTSD-8: A short PTSD inventory. Clinical Practice \& Epidemiology in Mental Health. 2010:6:101- 108.

[15] Mines, S., Veazey, C., Poling, S., Field, T. \& Bendell, D. (2020). PTSD symptoms, comorbid mental health and health behaviors during a COVID-19 lockdown. Annals of Psychiatry and Mental Health, 8(3), 1155-1159.

[16] Field, T., Poling, S., Mines, S., Diego, M., Bendell, D. \& Veazey, C. (2020). Boredom and psychological problems during a COVID-19 lockdown. Archives of Health Science, 1-8.

[17] Field, T., Poling, S., Mines, S., Bendell, D. \& Veazey, C. (2020) Exercise during a COVID-19 lockdown. Journal of Community Medicine and Public Health, 4 (3), 196-202.

[18] Field, T., Poling, S., Mines, S., Bendell, D. \& Veazey, C. Social media during a COVID-19 lockdown. Social Media and Society. In review.

[19] Field young and living alone paper Field, T., Mines, S., Poling, S., Bendell, D. \& Veazey, C. (2020). Young, alone, and young alone during a COVID-19 lockdown. Mental Health and Clinical, 4 (4), 31-38.

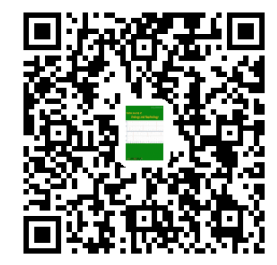

